\title{
Cardiovascular Risk Factors and Liver Transplantation. Minireview
}

\author{
A. Rossetto*, U. Baccarani, G.L. Adani, D. Lorenzin, A. C. Leo, L. Seriau, S. Bidinost, C. Comuzzi and V. Bresadola \\ Department of Surgery and Transplantation, University Hospital of Udine, Udine, Italy
}

\section{Introduction}

In the last decades, the survival of liver transplanted patients and grafts have had a great improvement due to many factors. Careful preoperative evaluation in transplant recipients, experience and a multidisciplinary approach have, without any doubt, a major role in the selection of candidates and in the diagnosis and treatment of preoperative complications. Moreover, with the introduction of new generation immunosuppressive drugs and careful pharmacological monitoring, both the episodes of acute rejection and the toxic effects have been minimized [1].

In consequence, since the graft and the patient post-transplant survival has been improved, the transplanted population has started showing long term medical complications.

Besides the risk correlated with graft rejection, the transplanted population has an increased risk of developing many malignancies. A number of hematological diseases, skin cancer, and gastrointestinal tumors seemsto recognizea possibletriggerfactorinimmunosuppressive drugs and in the patient's immunological status and serological status, with viral infections quite common in immunosuppressed patients. As for frequency, after these two groups of complications, cardiovascular diseases are the third cause of death in the transplanted population [1].

Eligibility to liver transplant, once based on Child Pugh system, has been regulated since a few years by MELD score (Model for End-Stage Liver Disease), an index of survival probability of the end stage liver disease [2]. This score is obtained on three variables: INR, creatinine and bilirubin. Due to the well known inadequate number of available grafts, if compared to patients requiring liver transplantation, and to the risk of mortality while in the waiting list, this score supplies a priority system, based on the severity of the disease, for the organ allocation. The cut off value normally considered for eligibility to liver transplantation is a MELD score $\geq 15$. Since the evaluation of the MELD score is obtained with this mathematical formula:

$10\{0.957 \operatorname{Ln}($ Serum Creatinine $)+0.378 \operatorname{Ln}($ Serum Bilirubin $)+1.12$ $\operatorname{Ln}(\mathrm{INR})+0.643\}$

Eligibility to transplantation undergoes the parameter of severity of the disease, selecting the population of patients with generally worse clinical conditions. Moreover, the median age of the patients waiting for transplantation seems to be higher, essentially due to the improvement in the antiviral therapies and the better medical treatment in the hepatological patients; for these reasons, the impairment of the liver function resulting in an eligible score can be delayed, with a longer conservative treatment and an older age of the patients who undergo liver transplantation.

Like in general population, the risk of developing cardiovascular disease increases with age, but liver disease by itself is often related to cardiovascular disease or higher cardiovascular risk factors $[2,3]$. Therefore, an accurate cardiovascular risk assessment of these patients, besides the whole pretransplant evaluation, is essential, both for the increased risk of cardiovascular diseases after liver transplant and for the cardiovascular risk assessment of the pre-transplant condition.

\section{Pretransplant Cardiovascular Risk}

Advanced hepatic liver disease is responsible for many changes in the physiology and biochemistry of the cardiovascular system, affecting contractility, heart rate, conduction and repolarization [4-6].

Cirrhotic cardiomyopathy is a pathological condition characterized by an increased cardiac output, impaired ventricular response to stress, decreased beta-agonist transduction, increased circulating inflammatory mediators with cardio depressant effect, alteration in repolarization, low systemic vascular resistance and bradycardia, altered function of muscarinic function and beta adrenergic stimulatory system, heart cell membrane abnormalities due to altered membrane fluidity and a modified calcium concentration, an overproduction of nitric oxide and cardio depressant effects of an increased level of carbon monoxide [7].

Both the systolic and diastolic functions seem to be damaged: the impairment of the systolic function, even if in cirrhotic cardiomyopathy the cardiac output is high, can be revealed by stress test, and can be caused by a reduced cardiovascular reactivity during exercise; the impairment of the diastolic function is determined by fibrosis, myocardial hypertrophy and sub endothelial edema resulting in an impairment in the compliance and relaxation [4-6].

Abnormalities at the ECG, such electromechanical dissociation which results in a prolongation of the QT interval, and chronotropic incompetence given by an impaired response to beta stimulation, are very common in cirrhosis and seem to be mainly related to portal hypertension and porto systemic shunting, but also to alterations of the heart rate with a central hypovolemia, loss of renal excretion of water and sodium and an altered baroreflex sensitivity contributing to impair the cardiovascular system $[4-6,8]$.

These conditions can lead to a higher risk of torsade de pointes, rhythm disturbances but also to the inability to develop physiologic tachycardia when required.

Some liver diseases seem to show a correlation also on the coronary blood flow. Diffuse but undetectable coronary atherosclerosis, reduced coronary micro vascular bed and impaired endothelium function are reported in non alcoholic fatty liver disease (NAFLD) $[9,10]$.

Pre-transplant diabetes is another cardiovascular risk factor, and, in liver diseases, the possibility to find an insulin resistance can be related to the pathogenesis of liver disease (NAFLD), where the deregulation of fat metabolism causes an overproduction of very low density proteins involved in the metabolic syndrome, and, after the initial over activity with hyperinsulinemia, there is an impairment of islet beta cells; on the

*Corresponding author: A. Rossetto, Department of Surgery and Transplantation, University Hospital of Udine, Udine, Italy, E-mail: rossettoannaar@libero.it

Received October 15, 2011; Accepted January 11, 2012; Published January 11 2012

Citation: Rossetto A, Baccarani U, Adani GL, Lorenzin D, Leo AC, et al. (2012) Cardiovascular Risk Factors and Liver Transplantation. Minireview. J Transplant Technol Res 2:109. doi:10.4172/2161-0991.1000109

Copyright: (c) 2012 Rossetto A, et al. This is an open-access article distributed under the terms of the Creative Commons Attribution License, which permits unrestricted use, distribution, and reproduction in any medium, provided the original author and source are credited. 
other hand, the liver glycogenogenesis and glycogenolysis pathways for the regulation of carbohydrate metabolism can be impaired due to the hepatic disease itself [10-17].

Renal dysfunction is not uncommon (microalbuminuria, hepatorenal syndrome), and also pulmonary heart diseases have to be considered such as hepatopulmonary syndrome where a mismatch between ventilation and perfusion is involved, with hypoxemia due to an excess of perfusion because of abnormal intrapulmonary vascular dilation $[5,9,11,16]$.

Porto pulmonary hypertension is a condition where pulmonary arterial hypertension is associated with liver disease or portal hypertension due to many factors such as hyperdinamic circulation, release of mediators from the congested bowel because of splancnic overload, leading to vasoconstriction and remodeling of the lung vascular endothelium [16].

The American Association for the Study of Liver Diseases [2] recommends a rigorous pre-operative Assessment (Figure 1).

\section{Post Transplant Cardiovascular Risk}

Patients who undergo liver transplantation have an around doubled risk of developing cardiovascular disease if compared to non transplanted population. To investigate the risk of such complications, multiple risk factors have to be added up. On one hand, there are all the risk factors of the general population such as advanced age, male gender, smoking history, high body mass index, pre-transplant diabetes mellitus, and marital status. On the other hand, some peculiar risks related to the etiology of the underlying liver cirrhosis have to be considered, since liver disease caused by criptogenetic, alcohol and hepatitis $\mathrm{C}$ seems to be related to an increased cardiovascular risk. Moreover, after transplantation, a new physiological condition arises from the hemodynamic, biochemical and drug related standpoint. The vasodilatation existing in the cirrhotic patient has been solved and a systemic vasoconstriction is quite often present in the transplanted patient. Many factors seem to be involved in such modification of the systemic resistances but a definitely pathogenesis is still under debate. The renin-angiotensin system seems to be involved but also the effect of endothelin, which seems to be higher than normal after LT, with its vasoconstrictor effect, could determine the increase of the arterial pressure as well as the arterial stiffness which is clearly related to high pressure. Anyway, arterial hypertension before transplant seems to be a factor contributing the post transplant development of arterial hypertension $[1,18,19]$.

The metabolic syndrome is apparently another risk factor, quite common in transplanted patient. In liver cirrhosis the patient generally suffers from a hyper metabolic condition and insulin resistance is very common. The normalization of the metabolic status, and the change induced in the metabolism of lipoproteins, in absence or combined with insulin resistance or diabetes due to the pancreas cells function impairment, can explain some effects of the post-transplant status while, the underlying liver disease itself (HCV infection and relapse) seems to be related to diabetes, affecting the insulin pathway and directly affecting the pancreatic beta cells [20-23].

A major role in the cardiovascular risk factors lies in the irreplaceable use of immunosuppressive drugs [1,18,24]. Immunosuppressive drugs are related to arterial hypertension and, especially calcineurin inhibitors, have turned out to affect the vascular bed causing endothelial dysfunction. Besides their direct effect on the vascular endothelium, they also affect the vascular smooth muscle cells and have effects on the release and production of nitric oxide and endothelin. The renal function is impaired because of the nephrotoxic effects of many of these drugs which cause an impairment of the glomerular filtration

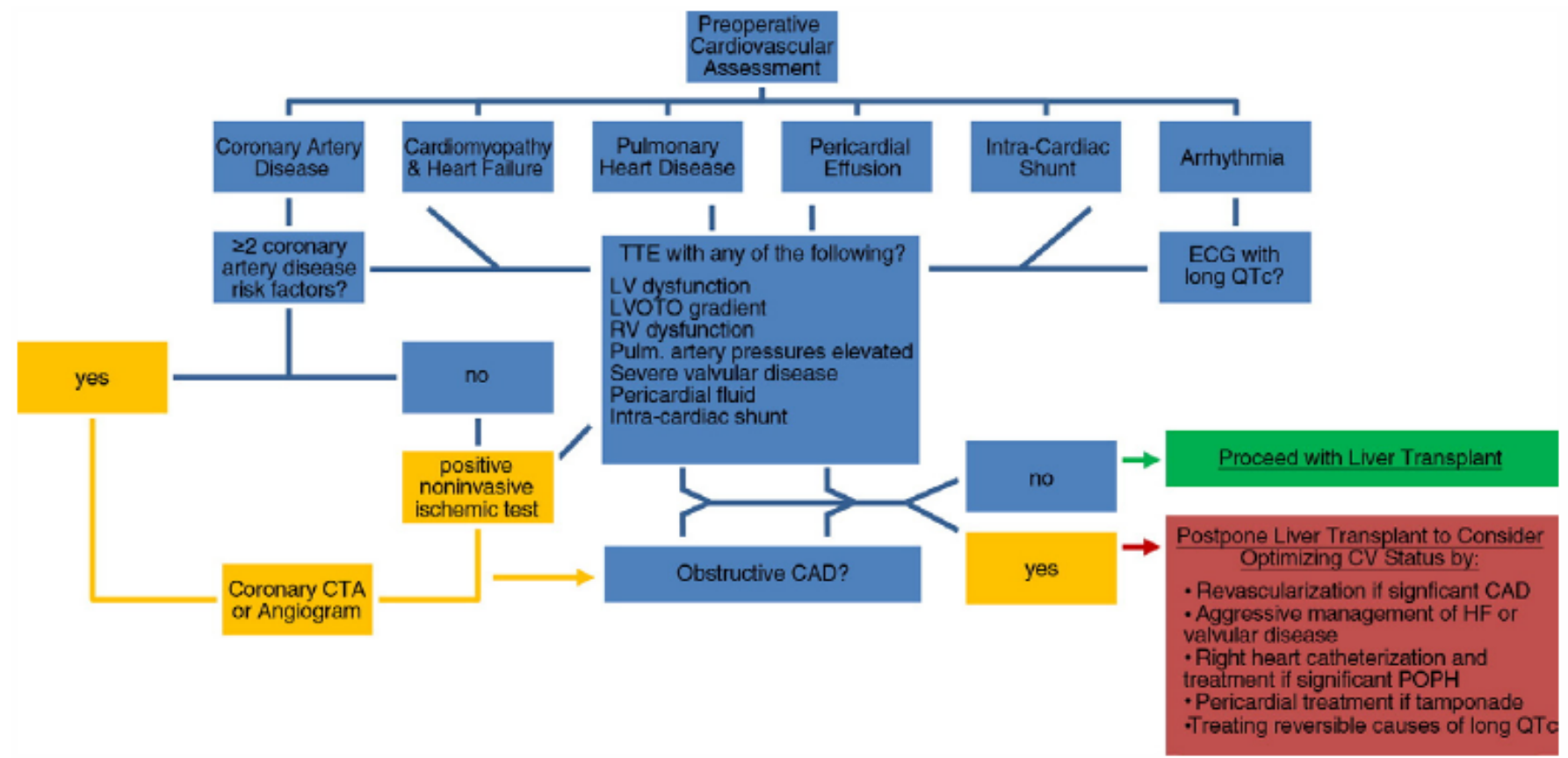

$\mathrm{CAD}=$ soronary artery disease $; \mathrm{CTA}=$ comuted tomography; $\mathrm{ECG}=$ electrocardiogram HF = heart failure; LV = left ventricular; LVOTO = left ventricular outflow tract ovstruction; POPH = portopulmonary hypertension; Pulm. = pulmonary; QTc = corrected QT interval; RV right ventricular; TTE = transthoracic echocardiogarphy

Figure 1: Suggested Srrategy for Pre-Operative Caddiac Assessment of Liver Transplant Candidates. 
rate and side effects on arterial pressure. Immunosuppressive drugs have also a pancreatic beta cells toxicity leading to a decrease in the production and secretion of insulin and an increased risk of diabetes [18]. This condition by itself impairs the micro vascular bed and renal function. These drugs, especially M-tor inhibitors, affect the lipid metabolism, with the development of high levels of cholesterol and triglyceride concentration requiring lipid-lowering drugs. This side effect seems to be due to a decreased drug related bile acid synthesis but also to the agonist effects of some immunosuppressive drugs with low density lipoprotein cholesterol receptors, with a higher quantity of circulating low density lipoproteins. These side effects cause a further impairing in the vascular bed status, with effects of stiffness on the vascular walls. Also the steroids, frequently used in combination with immunosuppressive drugs or as treatment of acute rejection, have their well known effects on the vascular system, causing vasoconstriction, arterial hypertension, truncal fat deposition, and on the glucydic metabolism, causing a decreased insulin production, and impairing the peripheral glucose utilization, resulting in an insulin resistance or diabetes $[1,18,25,26]$.

\section{Immunosuppression and Cardiovascular Risk Factors}

Transplantation soared in the 1980s thanks to the introduction of a new immunosuppressive drug: Cyclosporine. Before that, survival after liver transplantation was strongly impaired by the common onset of rejection and graft loss, when the immunosuppressive strategies were poor and crude such as the whole-body x-radiation at the very beginning (1960s) and azathioprine, prendisolone and antilymphocytes antibodies about ten years later [27].

The experience in liver transplant shows that liver transplant recipients develop a lower rate of rejection if compared to other organs (heart, kidney) possibly because a form of microchimerism due to a large number of donor's cells within the allograft. Also the production by the liver of soluble donor MHC class 1 molecules has been mentioned to explain this resistance to rejection in liver transplantation. The size and regenerative properties of liver can also play a role [27,28]. Nevertheless, although the liver is less prone to rejection then other organs, the immunosuppressive regimen remains mandatory after liver transplantation. The selection of immunosuppressive drugs is not universal but has to be done considering the pretransplant history and the medical conditions, considering for example pretransplant poor renal function, diabetes or hepatocellular carcinoma. Therefore a careful balance between pros and cons is essential.

At present, calcineurin inhibitors are the most largely used immunosuppressive drugs for liver transplantation, with well known side effects such as neurotoxicity, nephrotoxicity, hypertension, increased risk of death due to cardiovascular risk factors, gingival hyperplasia, hirsutism and diabetes.

Cyclosporine, the oldest one, is derived from the fungus Tolypocladium inflatum and is a polypeptide of 11 amino acids. Tacrolimus, the most recent one, is derived from Streptomyces tsukabaensis and is a macrolide compound.

Cyclosporine inhibits interleukin 2 gene transcription, binding cyclophilin, inhibiting the calcium/calmodulin phosphatase dependent calcineurin complex. It causes a dephosphorilation of activated $\mathrm{T}$ cell which is important for the transcription of cytokines for the activation of T cells, while Tacrolimus, from the same group of immunosuppressive drugs and with a similar mechanism of action, inhibits calcineurin binding to another specific immunophilin, FK binding protein 12 [2729].
These drugs seem to have complex activities besides immunosuppression, causing endothelial dysfunction and more specifically a decrease in the production of the vasodilator nitric oxide (NO) by endothelial NO synthase (eNOS), affecting the vasodilator function by negatively altering endothelial intracellular $\mathrm{Ca} 2+$ and eNOS phosphorylation. Ca2+ concentration is altered by intracellular $\mathrm{Ca} 2+$ leak and decreased agonist-induced intracellular $\mathrm{Ca} 2+$ release which negatively affects eNOS phosphorylation, NO production, and endothelium-dependent dilatation. The precise mechanisms leading to hyperlipidemia are not completely known. There are contributing factors such as corticosteroids use and obesity. Anyway cyclosporine is related to an increase of VLDL and LDL while Tacrolimus seems to be characterized by VLDL increase alone. The mechanisms which underlie these side effects seem to be the increase in free intracellular cholesterol levels due to an impaired cholesterol esterification, but also the activation of the transcription factor responsible for the expression of lipid related genes. Oxidative processes may also underlie the atherosclerotic effect.

The nephrotoxic effects are well known, and they can, by themselves, be related to the development of arterial hypertension. The mechanism, beside a possible involvement of the tubular epithelium, seems to be more strongly related to vascular alteration of the afferent arterioles, with consequent ions alteration and hyperkaliemia [28-30].

The arteriolar vasoconstriction and renal ischemia are related to an imbalance between the vasoactive messengers (endothelin-1 and tromboxane A2), the dysregulation of nitric-oxide formation and the renin-angiotensin system. Besides these vascular effects altering the renal control of arterial pressure, the nephrotoxic direct effects should also be mentioned, with tubular cell apoptosis and necrosis and effects on the cell cycle compromising the proliferation capacity and accelerating cell aging, possibly mediated by an oxidative stress caused by reactive oxygen species (ROS) and lipid peroxides. This effect has been demonstrated as an abnormality in permeability of the mitochondria causing an isometric vacuolization resulting in the presence of giant mitochondria. Thus, the endoplasmic reticulum undergoes enlargement and the protein synthesis is affected. Cyclosporine can act as an endoplasmic reticulum stress inducer causing epithelial phenotypic changes leading to nephrotoxicity [3034].

Endoplasmic reticulum stress seems to play a major role in many diseases such as atherosclerosis, Alzheimer, diabetes and inflammatory bowel diseases, leading the unfolded protein response to an adaptative response [35,36].

The above mentioned stress is involved in post transplant diabetes, promoting insulin resistance and cell death. Insulin secernig cells undergo protein synthesis stress and are very sensitive to any status causing accumulation of anomalous proteins. The pathophysiology of pancreatic beta cells damage during treatment with Tacrolimus and Cyclosporine is still matter of debate, but it seems related to the endoplasmic reticulum stress which modifies cells vitality, since in animal models treated with calcineurin inhibitors, nuclear inclusions, dilatation in cistern of the granulous endoplasmic reticulum with degranulation and degeneration of pancreatic beta islets have been shown $[35,36]$.

Steroids are often used in immunosuppressive therapeutic schemes in the perioperative period, both intraoperatively and after transplantation, for a period ranging from 3 to 6 months for liver transplantation. Moreover, at the possible onset of acute rejection, bolus of steroids is administered. The steroid immunosuppressive 
function derives from the inactivation of the response of lymphocytes and macrophages by inhibiting the production of cytokines, but also suppressing antigens and stimulating the migration of $\mathrm{T}$ cells to the lymphoid tissue [30].

A large body of evidence supports the theory that steroids induce an imbalance between vasoconstriction and vasodilatation, favoring vasoconstriction, resulting in arterial hypertension.

The increased vasoconstriction is mediated by several mechanisms. In large part vasoconstriction is mediated by the increased endothelin-1 synthesis and secretion, increased erythropoietin levels by the increased level of cytosolic calcium, increased sympathetic activity that is mediated by the increase of Beta1-adrenergic receptor expression and increased synthesis of catecholamines, by the increased expression of various enzymes involved in the catecholamines biosynthesis, including tyrosine hydroxilase and phenyl ethanolamine N-methyltransferase and altered availability of Alfa1-adrenergic receptors in vascular smooth muscles, leading to an increased vascular reactivity, pressure responsiveness and arterial hypertension. The vasoconstriction and hypertension induced by steroids are also mediated through enhanced synthesis and action of vasoactive substances and their receptors, including neuro peptide Y (NPY), arginine vasopressin (AVP) and atrial natriuretic peptide (ANP).

Another interesting issue is the role of the renin-angiotensin system activation in the development of steroids induced hypertension. Steroids act directly at the liver site, enhancing the synthesis of angiotensinogen $[29,37]$.

On the other hand, steroids negatively affect various vasodilatory systems causing nitric oxide (NO) deficiency through a range of negative influences on the NO biosynthetic pathways involving alteration in the activity and expression of NO synthase, decreased availability of tetrahydrobiopterin (BH4) and decreased $\mathrm{NO}$ precursor, L-arginine. Moreover, steroids affect the production of other vasodilatatory substances as prostacyclin, prostaglandin E2 and kallikrein.

Steroids induce insulin resistance in skeletal muscle by directly interfering with the insulin signaling cascade. The same effect is produced also in hepatic cells and thus, endogenous glucose production is increased $[28,29,37]$.

Steroids appear to have a direct causal effect relationship with cardiovascular disease depending on dose, duration, cumulative dose of exposure and route of administration. The increased risk is mediated through the induction of several risk factors for cardiovascular disease.

Since the late 1990s other immunosuppressive drugs have been introduced into the world of transplantation: the group of $\mathrm{m}$-Tor inhibitors (Sirolimus and Everolimus) [27-29].

Sirolimus is derived from actinomycete Streptomyces hygroscopicus. It has a homologous structure if compared to Cyclosporine and Tacrolimus and it also binds to FK bindig proteins family (FKBP-12) which binds to mammalian targets of rapamycin (m-TOR), which has a kinase activity. Sirolimus plays its action on the signal transduction pathway, blocking the IL-2 and IL-5 induction of $\mathrm{B}$ and $\mathrm{T}$ cell proliferation. It inhibits the normal cell proliferation in response to IL-2. Everolimus is a macrolide derived from Sirolimus, with a similar mechanism of action, inhibiting the activation of immunophilin FKBP-12. Unlike Sirolimus, it has pharmacokinetics properties which make it easier to handle.

These drugs are particularly interesting and under debate. Based on their anti proliferative effects, they also seem to have anti-neoplastic effects based on the fact that they inhibit angiogenesis, inhibit cancer cells survival and also cancer stem cells survival [27-29].

They seem to have less nephrotoxic effects than calcineurininhibitors provided there is no pre-existing renal disease, basing their action on $\mathrm{m}$-Tor contrasting the renal fibrosis caused by TGF-beta. There's anyway a risk of proteinuria or of renal damage amplification in an already compromised renal function or in combination with calcineurin inhibitors (CNI).

They show interesting properties in promoting tolerance but Sirolimus is considered an unsafe drug for the $1^{\text {st }}$ month after transplantation for a higher risk of developing hepatic artery thrombosis and for a slower and more difficult wounds healing. Mouth ulcers and leg edema are common. It's strongly related to high risk of developing hyperlipidemia, which seems to be caused by an increased hepatic secretion of VLDL, increased hepatic synthesis of apoB100 and a reduction in the hepatic catabolism of LDL. The action of lipoprotein lipase is decreased and the expression of apoCIII and lipase in adipose tissue are increased. Anyway these drugs have effects on macrophages and antiproliferative effects which could protect from the cardiovascular risk of atherosclerosis [27-29,38,39].

Antimetabolites such as mycophenolate mofetil and mycophenolate sodium have immunosuppressive properties which were recognized in the 1990s although these drugs are older. Their action derive from the blockage of inosine- $5^{1}$-monophosphate dehydrogenase (IMPDH) resulting in the selective lymphocyte proliferation blockage. They have probably less cardiovascular side effects, mainly gastrointestinal disorders, bone marrow depression, and some infections. Their exclusive use is hardly ever considered due to their insufficient protection from acute rejection if not combined to $\mathrm{m}$-Tor or CNI. Yet their use anyway often allows a lower CNI or m-Tor dosage with side effects sparing properties [27-29,40].

Both polyclonal and monoclonal antibodies [anti-thymocites globulin (ATG), anti-lymphocyte globulin (ALG), monoclonal antibodies (OKT3, Campath, Basiliximab)] are also largely used in combination with CNI or m-Tor delayed introduction, as induction therapies, minimizing the side effects of CNI and $\mathrm{m}$-Tor inhibitors.

Other immunosuppressive drugs are currently undergoing trials such as Belatacept, which inhibits T cell activation binding CD80 and CD86, and looks promising, with less renal toxicity, and Efalizumab which inhibits T cell-APC stabilization and blocks lymphocyte adhesion to endothelial cells, with good results as for immunosuppressive properties but with an increased risk of onset of post-transplant lymphoproliferative disorder (PTLD) [27-29] (Table 1,2 and 3).

\section{Conclusions}

Besides the cardiovascular risk factors related to age, family disease and life habits, the transplant candidate has a particular hemodynamic, biochemical, cardiac and systemic condition depending on the hepatic disease. After transplantation, these para physiologic modifications suddenly change, with the onset of a new systemic condition, the appearance of organ damages not evident before, a new risk factors profile related to the new situation and to unavoidable life-saving drugs treatment.

For these reasons the pre-transplant cardiovascular evaluation and the post-transplant accurate monitoring followed by a careful choice of the immunosuppressive therapeutic regimen, drug level monitoring, educational efforts to ameliorate life style and risk factors are mandatory for a satisfactory outcome. 
Citation: Rossetto A, Baccarani U, Adani GL, Lorenzin D, Leo AC, et al. (2012) Cardiovascular Risk Factors and Liver Transplantation. Minireview. J Transplant Technol Res 2:109. doi:10.4172/2161-0991.1000109

\begin{tabular}{|c|c|}
\hline IMMUNOSUPPRESSIVE AGENT & MECHANISM OF ACTION \\
\hline CALCINEURIN INHIBITORS & Inhibit signal 2 trasduction via $\mathrm{T}$ cell receptor \\
\hline MAMMALIAN TARGET OF RAPAMYCIN INHIBITORS & Inhibit signal 3 trasduction via IL-2 receptor \\
\hline MYCOPHENOLIC ACID & Inhibits purine and DNA synthesis \\
\hline CORTICOSTEROIDS & $\begin{array}{l}\text { Inhibit cytokine transcription by antigen presenting cell; } \\
\text { Selective lysis of immature cortical thymocytes }\end{array}$ \\
\hline AntiCD3 monoclonal antibodies & Depletion and receptor modulation in T cell Interferes with signal 1 \\
\hline Antithymocyte globuline & $\begin{array}{l}\text { Depletion and receptor modulation in T cells } \\
\text { Interferes with signal } 1,2,3 \\
\text { Inhibits lymphocytes trafficking }\end{array}$ \\
\hline Anti IL-2 alpha chain receptor antibodies & Inhibit T cell proliferation to IL-2 \\
\hline Anti-CD52 monoclonal antibodies & Cause depletion of thymocytes, $\mathrm{T}$ cells, B cells and monocytes \\
\hline
\end{tabular}

Table 1: Immunosuppressive Drugs and Mechanism of Action.

\begin{tabular}{|c|c|}
\hline IMMUNOSUPPRESSIVE AGENTS & SIDE EFFECTS \\
\hline CALCINEURIN INHIBITORS & Hypertension, renal toxicity, neurotoxicity, diabetes, dislipidemia, gingival hyperplasia, hirsutism. \\
\hline $\begin{array}{l}\text { MAMMALIAN TARGET OF RAPAMYCIN } \\
\text { INHIBITORS }\end{array}$ & $\begin{array}{l}\text { Dislipidemia, anemia, leucopenia, thrombocytopenia, hepatic artery thrombosis, wound dehiscence, aphtous ulcers, } \\
\text { arthralgia, proteinuria }\end{array}$ \\
\hline MYCOPHENOLIC ACID & Anorexia, abdominal pain, gastritis, diarrhea, neutropenia \\
\hline CORTICOSTEROIDS & $\begin{array}{l}\text { Hypertension, pancreatitis, peptic ulcer, osteoporosis, aseptic necrosis of femoral head, diabetes, hyperlipidemia, risk of } \\
\text { infections, difficult wound healing }\end{array}$ \\
\hline AntiCD3 monoclonal antibodies & Fever, hypotension, headache, aseptic meningitis, dyspnea, vomiting, diarrhea \\
\hline Antithymocyte globuline & Leucopenia, thrombocytopenia \\
\hline Anti IL-2 alpha chain receptor antibodies & Risk of acute rejection \\
\hline Anti-CD52 monoclonal antibodies & Risk of acute rejection \\
\hline
\end{tabular}

Table 2: Immunosuppressive Drugs and Side Effects.

\section{IMMUNOSUPPRESSIVE AGENT PATHOPHYSIOLOGY of CARDIOVASCULAR SIDE EFFECTS} \begin{tabular}{l|l} 
& $\begin{array}{l}\text { Endothelial dysfunction, decreased production of NO, altered concentration of Ca2+, impaired cholesterol esterification, increased } \\
\text { free intracellular cholesterol levels, oxidative processes, vascular alterations of the afferent arteriolas, ions alterations and }\end{array}$
\end{tabular} hyperkaliemia, tubular cell apoptosis and necrosis, endoplasmic reticulum stress leading to nephrotoxicity and pancreatic beta cells damage

\section{MAMMALIAN TARGET OF $\quad$ Contrast the renal fibrosis caused by TGF-beta}

RAPAMYCIN INHIBITORS Increased hepatic secretion of VLDL, increased hepatic synthesis of apoB100, reduction in the hepatic catabolism of LDL.

Decreased action of lipoprotein lipase, increased expression of apoCIII and lipase in adipose tissue.

\section{CORTICOSTEROIDS}

Imbalance between vasoconstriction and vasodilatation, increase synthesis and secretion of endothelin-1, increased erythropoietin levels, increased sympathetic activity, increased expression of vasoactive substances and altered function of receptors, nitric oxide deficiency, modified insulin signaling cascade, increased endogenous glucose production.

Table 3: Immunosuppressive Drugs and Pathophysiology of Cardiovascular Side Effects.

\section{References}

1. Rossetto A, Bitetto D, Bresadola V, Lorenzin D, Baccarani U, et al. (2010) Cardiovascular risk factors and immunosuppressive regimen after liver transplantation. Transplant Proc 42: 2576-2578.

2. Raval Z, Harinstein ME, Skaro Al, Erdogan A, DeWolf AM, et al. (2011)
Cardiovascular risk assessment of the liver transplant candidate. J Am Col Cardiol 58: 223-231

3. Aberg F, Jula A, Höckerstedt K, Isoniemi H (2010) Cardiovascular risk profile of patients with acute liver failure after liver transplantation when compared with the general population. Transplantation 89: 61-68. 
Citation: Rossetto A, Baccarani U, Adani GL, Lorenzin D, Leo AC, et al. (2012) Cardiovascular Risk Factors and Liver Transplantation. Minireview. J Transplant Technol Res 2:109. doi:10.4172/2161-0991.1000109

Page 6 of 6

4. Zardi EM, Abbate A, Zardi DM, Dobrina A, Margiotta D, et al. (2010) Cirrhotic cardiomyopathy. J Am Coll Cardiol 56: 539-549.

5. Lee RF, Glenn TK, Lee SS (2007) Cardiac dysfunction in cirrhosis. Best Pract Res Clin Gastroenterol 21: 125-140

6. Møller S, Henriksen JH (2010) Cirrhotic cardiomyopathy. J Hepatol 53: 179190.

7. Rockey DC (2003) Vascular mediators in the injured liver. Hepatology 37: 4-12.

8. Yilmaz Y, Kurt R, Yonal O, Polat N, Celikel CA, et al. (2010) Coronary flow reserve is impaired in patients with nonalcoholic fatty liver disease: association with liver fibrosis. Atherosclerosis 211: 182-186.

9. Mandell MS, Lindenfeld J, Tsou MY, Zimmerman M (2008) Cardiac evaluation of liver transplant candidates. World J Gastroenterol 14: 3445-3451.

10. Fon Tacer K, Rozman D (2011) Nonalcoholic Fatty liver disease: focus on lipoprotein and lipid deregulation. J Lipids 2011: 783976.

11. Garcia-Compean D, Jaquez-Quintana JO, Gonzalez-Gonzalez JA, MaldonadoGarza H (2009) Liver cirrhosis and diabetes: risk factors, pathophysiology, clinical implications and management. World J Gastroenterol 15: 280-288.

12. Parekh S, Anania FA (2007) Abnormal lipid and glucose metabolism in obesity: implications for nonalcoholic fatty liver disease. Gastroenterology 132: 2191 2207.

13. Petrides AS, Groop LC, Riely CA, DeFronzo RA (1991) Effect on physiologic hyperinsulinemia on glucose and lipid metabolism in cirrhosis. J Clin Invest 88 561-570.

14. Krok KL, Milwalla F, Maheshwari A, Rankin R, Thuluvath PJ (2009) Insulin resistance and microalbuminuria are associated with microvascular disease in patients with cirrhosis. Liver Transpl 15: 1036-1042.

15. García-Compean D, Jaquez-Quintana JO, Maldonado-Garza H (2009) Hepatogenous diabetes. Current views of an ancient problem. Ann Hepato I 8: $13-20$.

16. Hoeper MM, Krowka MJ, Strassburg CP (2004) Portopulmonary hypertension and hepatopulmonary syndrome. Lancet 363: 1461-1468.

17. Laish I, Braun M, Mor E, Sulkes J, Harif Y, et al. (2011) Metabolic syndrome in liver transplant recipients: prevalence, risk factors, and association with cardiovascular events. Liver Transpl 17: 15-22.

18. Tepperman E, Ramzy D, Prodger J, Sheshgiri R, Badiwala M, et al. (2010) Surgical biology for the clinician: vascular effects of immunosuppression. Can J Surg 53: 57-63.

19. Neal DA, Brown MJ, Wilkinson IB, Alexander GJ (2005) Mechanisms of hypertension after liver transplantation. Transplantation 79: 935-940.

20. Watt KD, Charlton MR (2010) Metabolic syndrome and liver transplantation: a review and guide to management. J Hepatol 53: 199-206.

21. Sorice GP, Muscogiuri G, Mezza T, Prioletta A, Giaccari A (2011) Metabolic syndrome in transplant patients: an academic or a health burden? Transplant Proc 43: 313-317.

22. Pagadala M, Dasarathy S, Eghtesad B, McCullough AJ (2009) Posttransplan metabolic syndrome: an epidemic waiting to happen. Liver Transpl 15: 1662 1670.

23. Ruiz-Rebollo ML, Sánchez-Antolín G, García-Pajares F, Fernández-Orcajo P, González-Sagrado M, et al. (2010) Risk of development of the metabolic syndrome after orthotopic liver transplantation. Transplant Proc 42: 663-665.

24. Pérez MJ, García DM, Taybi BJ, Daga JA, Rey JM, et al. (2011) Cardiovascula risk factors after liver transplantation: analysis of related factors. Transplant Proc 43: 739-741.

25. Farge D, Julien J (1998) Effects of transplantation on the renin angiotensin system (RAS). J Hum Hypertens 12: 827-832.

26. Bahirwani R, Reddy KR (2009) Outcomes after liver transplantation: chronic kidney disease. Liver Transpl 2: S70-S74.

27. Geissler EK, Schlitt HJ (2009) Immunosuppression for liver transplantation. Gut 58: 452-463.

28. Pillai AA, Levitsky $J$ (2009) Overview of immunosuppression in liver transplantation. Worl J Gastroentol 15: 4225-4233.

29. Mukherjee S, Mukherjee U (2009) A comprehensive review of immunosuppression used for liver transplantation. J Transplant 2009: 701464.

30. Kockx M, Jessup W, Kritharides L (2010) Cyclosporin A and atherosclerosisCellular pathways in atherogenesis. Pharmacol Ther 128: 106-118.

31. Halloran PF (2001) Mechanism of action of the calcineurin inhibitors. Transplant Proc 33: 3067-3069

32. Chapman JR (2011) Chronic Calcineurin Inhibitor Nephrotoxicity-Lest We Forget. Am J Transplant 11: 693-697.

33. Lamoureux F, Mestre E, Essig M, Sauvage FL, Marquet P, et al. (2011) Quantitative proteomic analysis of cyclosporine-induced toxicity in a human kidney cell line and comparison with tacrolimus. J Proteomics 75: 677-694.

34. Pallet N, Fougeray S, Beaune P, Legendre C, Thervet E, et al. (2009) Endoplasmic Reticulum Stress: An Unrecognized Actor in Solid Organ Transplantation. Transplantation 88: 605-613.

35. Pallet N, Bouvier N, Beaune P, Legendre C, Anglicheau D, et al. (2010) Involvement of endoplasmic reticulum stress in solid organ transplantation. Med Sci (Paris) 26: 397-403.

36. Pham PT, Pham PM, Pham SV, Pham PA, Pham PC (2011) New onse diabetes after transplantation (NODAT): an overview. Diabetes Metab Syndr Obes 4: 175-186.

37. Peppa M, Krania M, Raptis SA (2011) Hypertension and other morbidities with Cushing's syndrome associated with corticosteroids: a review. Integr Blood Press Control 4: 7-16.

38. Gabardi S, Baroletti S (2010) Everolimus: A proliferation signal inhibito with clinical applications in organ transplantation, oncology and cardiology. Phramacotherapy 30: 1044-1056.

39. Webster AC, Lee VW, Chapman JR, Craig JC (2006) Target of rapamycin inhibitors (Sirolimus and Everolimus) for primary immunosuppression of kidney transplant recipients: a systematic review and meta-analysis of randomized trials. Transplantation 81: 1234-1248.

40. Allison AC, Eugui EM (2000) Mycophenolate mofetil and its mechanism of action. Immunopharmacology 47: 85-118.

41. Mancia G, De Backer G, Dominiczak A, Cifkova R, Fagard R, et al. (2007) 2007 Guidelines for the management of arterial hypertension: The Task Force for the Management of Arterial Hypertension of the European Society of Hypertension (ESH) and of the European Society of Cardiology (ESC). Eur Heart J 28: 1462 1536.

42. Wilkinson A, Davidson J, Dotta F, Home PD, Keown P, et al. (2005) Guidelines for the treatment and management of new-onset diabetes after transplantation. Clin Transplant 19: 291-298. 